Published online 2017 April 13.

Abstract

\title{
Breast Cancer Screening with MRI
}

\section{Thomas. Helbich ${ }^{1,}$}

${ }^{1}$ Department of Biomedical Imaging and Image guided Therapy, Medical University of Vienna

"Corresponding author: Thomas. Helbich, Department of Biomedical Imaging and Image guided Therapy, Medical University of Vienna. E-mail: thomas.helbich@meduniwien.ac.at

Received 2016 December 21; Accepted 2017 February 08.

\begin{abstract}
The sensitivity and specificity of mammography are limited in highly fibroglandular breasts (ACR density C and D). Digital mammography provides increased sensitivity in young women and those with moderately dense breasts, and digital three-dimensional mammography (Tomosynthesis) promises further improvement. For women with the densest breasts, however, radiography is unlikely to be the optimum solution. MRI, although not affected by breast density, is expensive and access is often limited. Nevertheless, enthusiasm for the use of MRI as a screening modality is growing because MRI demonstrated to be the most sensitive test and cancer yield is significant higher in comparison to any other imaging technology. This has been demonstrated in women at high and average risk. In comparison to mammography the specificity is lower, but technical developments have given rise to sharper, more informative images. These improvements foster the use of MRI. Different trials have been preformed and promising results have been reported. This talk will focus on benefits, harms, and cost-effectiveness of supplemental MRI based breast cancer screening.
\end{abstract}

This is an abstract presented in the 33rd Iranian congress of radiology (ICR) and the 15th congress of Iranian radiographic science association (IRSA). 